1365

\section{DOES THE PAIN SCALE TYPE INFLUENCE THE ASSESSED PAIN INTENSITY IN PAINFUL SICKLE CELL CRISIS IN THE PEDIATRIC EMERGENCY DEPARTMENT?}

E. Courtois ${ }^{1}$, L. Al Lawati ${ }^{1}$, L. Tostivint ${ }^{1}$, B. Quinet ${ }^{2}$, E. Lesprit ${ }^{2}$, C. Batard ${ }^{1}$, B. Castello ${ }^{1}$, C. Bouvatier ${ }^{1}$, C. Velly ${ }^{1}$, N. De Suremain ${ }^{1}$, R. Carbajal ${ }^{1}$

${ }^{1}$ Pediatric Emergency Department, Armand Trousseau University Hospital, AP-HP, ${ }^{2}$ General Pediatrics Department, Armand Trousseau University Hospital, AP-HP, Paris, France

The Visual Analog Scale (VAS) is the gold-standard for pain assessment. Since it cannot always be used, staffs often assess pain with other scales in children with Painful Sickle Cell Crises-(PSCC) visiting the Emergency Department-(ED).

Objectives: To compare the initial severity of PSCC in children presenting to the ED according to the used pain scale.

Methods: Retrospective study of all one-year visits to a pediatric ED for a PSCC. ED policy allowed triage nurses to use the pain scale they deemed the most suitable for each child.

Results: From May 2008 to April 2009, 189 visits concerning 87 children were registered. In 149 (79\%) visits, an initial pain score was recorded. Triage nurses assessed pain using four scales. The mean (SD) age was $12.3(3.1)$ years for $97(65.1 \%)$ visits of children assessed with a 0-10 VAS or Numerical Rating Scale (VAS-NRS group), 5.2 (3.4) years for $45(30 \%)$ assessments with 0-10 Faces Scale, 4.2 (4.0) for 5 assessments with 0-15 EVENDOL, and 2.2 (2.2) for 2 assessments with 0-8 Objective Pain Scale (OPS); ANOVA $p<0.001$. In order to uniform all the assessment data, EVENDOL and OPS were transformed into a $0-10$ scale. The median (interquartile) uniformed pain scores were 7.0 (6.08.0) for VAS-NRS, 4.0 (4.0-6.0) for Faces Scale, 4.0 (1.3-6.3) for EVENDOL, and 7.5 (5.0-10) for OPS; Kruskal-Wallis $p<0.001$.

Conclusions Severity of pain in children with PSCC varied with the scale used. It is postulated that the Faces or the EVENDOL scale underestimate pain in PSCC.

\section{THE EVOLUTION OF PAIN DURING HOSPITALIZATION OF CHILDREN WITH PAINFUL SICKLE CELL CRISES}

L. Al Lawati ${ }^{1}$, E. Courtois ${ }^{1}$, L. Tostivint ${ }^{1}$, E. Lesprit ${ }^{2}$, B. Quinet ${ }^{2}$, C. Galeotti ${ }^{1}$, J.-B. Armengaud ${ }^{1}$, R. Carbajal ${ }^{1}$

${ }^{1}$ Pediatric Emergency Department, Armand Trousseau University Hospital, AP-HP, ${ }^{2}$ General

Pediatrics Department, Armand Trousseau University Hospital, AP-HP, Paris, France

Relieving pain effectively in children with painful sickle cell crisis (PSCC) is a challenging clinical problem. Notwithstanding analgesic treatment, some hospitalized children for a PSCC seem to continue in pain.

Objectives: To describe the evolution of pain in hospitalized children receiving analgesics for a PSCC.

Methods: Retrospective study of all one-year hospitalizations for PSCC in a pediatric university hospital. Pain assessments were retrieved from patients' records. The minimum, mean and maximum pain scores per day of hospitalization were determined for each patient.

Results: From May 2008 to April 2009, 156 admissions for PSCC occurred. The median (interquartile; extremes) age of admitted patients was 9.9 (5.1-13.5; 0.3-18.3) years. The mean (SD; extremes) duration of hospitalization was 3 (2.8; 0-17) days. The mean (SD; extremes) pain score on arrival to the ward was 3.9 (3.3; 0-10). During the hospitalization period the intensity of pain decreased progressively from day 1 to day 7 , thereafter there was an increase in the intensity of pain followed by a decrease on day 14. Figure shows the distribution of maximum pain scores for each day of hospitalization.

Conclusion: Daily maximum pain scores are high $(>5 / 10)$ for about a quarter of children during the first 6 days of hospitalization for PSCC notwithstanding analgesic treatment. Improvement of current analgesic protocols seems necessary. 